\title{
Banach 空间的球覆盖性质
}

献给吴从炘教授 85 华诞

\section{张文}

厦门大学数学科学学院, 厦门 361005

E-mail: wenzhang@xmu.edu.cn

收稿日期: 2020-03-02; 接受日期: 2020-04-26; 网络出版日期: 2020-11-26

国家自然科学基金 (批准号: 11731010) 资助项目

摘要 如果 Banach 空间 $X$ 的单位球面可以被可数个不包含原点的球所构成的球族所覆盖, 则称 $X$ 具有球覆盖性质. 本文对 Banach 空间的球覆盖性质这个研究方向的发展进行简要回顾, 同时也给出 一些新的相关研究成果.

关键词 Banach 空间 球覆盖性质 次微分映射
MSC (2010) 主题分类 $46 \mathrm{~B} 20$

\section{1 引言}

赋范空间中单位球和单位球面的几何性质和拓扑性质在 Banach 空间几何学中占有非常重要的地 位. Banach 空间几何学, 可以说就是与 Banach 空间单位球和单位球面有关的学科. 几乎所有的几何 概念都是通过单位球来定义的, 如 Banach 空间的凸性、光滑性和自反性等. 不仅如此, 还有许多研究 课题都是与 Banach 空间子集的球表示问题有关的, 如 Mazur 交性质、装球问题、非紧性测度和球拓 扑问题等 ${ }^{[1-8]}$. 这些课题从提出开始就引起了许多数学家的关注, 经过前人孜孜不倦的努力, 这些问题 的研究已经取得了许多重要的成果, 这些结果往往又对 Banach 空间几何性质的深入研究有着不可或 缺的作用, 可见球族行为的鬼力是令人惊叹的.

关于 Mazur 交性质, 最早是 Mazur 注意到 Euclid 空间的这一性质: 每个有界闭凸集可以表示成 闭球的交, 于是, 线性赋范空间的这种性质被命名为 Mazur 交性质. 我们容易知道, 有界闭凸集 $C$ 是 闭球的交当且仅当对任意不属于 $C$ 的 $x$, 存在包含 $C$ 的闭球但不包含 $x$, 所以, Mazur 交性质可以看 成是球分离性质, 比经典的超平面分离性要强. 在之后的关于 Mazur 交性质的研究中, 人们比较关注 的问题则是 Mazur 交性质特征的刻画.

装球问题 (spherepacking) 是指把不相交的相同大小的球排满整个空间. 一般来说, 这个空间指的 是 3 维空间. 但是装球问题也可以推广到二维空间 (此时 “球” 就是指圆)、 $n$ 维空间 (此时 “球” 是指 
超球面) 和非 Euclid 空间 (如双曲空间). 经典的装球问题是寻找一种排列使得球以尽可能大的比例 占据整个空间, 这个装满空间的比例称之为排列的密度. 由于排列的密度取决于体积的大小, 所以, 这 个问题通常都是在一个充分大体积的立体内, 最大化其平均或者渐近密度. 一个正规的排列是指球心 都组成一个称之为格的整齐的模式. 如果球心没有排列成一个格, 这种排列称之为非正规的排列. 正 规排列往往比非正规排列更容易掌握, 这是由于对称性使得它们易于被分类和测量其密度.

1930 年, Kuratowski ${ }^{[9]}$ 首次引入了非紧性测度的概念, 并且研究了其相关性质和拓扑结构. 1955 年, Darbo ${ }^{[10]}$ 首次利用 Kuratowski 非紧性测度给出著名的 Darbo 不动点定理. 设 $(X, \rho)$ 为度量空 间, $\mathcal{D}(X)$ 为 $X$ 上所有非空有界子集全体, 对于任意 $D \in \mathcal{D}(X)$, 其 Kuratowski 非紧性测度 $\alpha(D)$ 定 义为 $\inf \left\{d>0: D \subset \bigcup_{j \in F} E_{j}, F \subset \mathbb{N}, F^{\sharp}<\infty\right.$ 且 $\left.d\left(E_{j}\right) \leqslant d\right\}$, 其中 $d\left(E_{j}\right)$ 表示 $E_{j} \subset X$ 的直径, 即 $d\left(E_{j}\right)=\sup \left\{\rho(x, y): x, y \in E_{j}\right\}$. 由定义可知 $\alpha(D) \leqslant d(D)$. Kuratowski 非紧性测度也称为集合非紧性 测度, 用来衡量度量空间中的一个有界集与紧集之间的 “距离”. 1957 年, Goldenstein 等 ${ }^{[11]}$ 在此基础 上引入了 Hausdorff 非紧性测度, 也称为球非紧性测度, $\beta(D)$ 的球非紧性测度则定义为

$$
\inf \left\{d>0: D \subset \bigcup_{j \in F} \bar{B}(x, r), F \subset \mathbb{N}, F^{\sharp}<\infty\right\},
$$

其中 $\bar{B}(x, r)$ 表示 $X$ 中以 $x$ 为圆心、 $r$ 为半径的闭球. 非紧性测度理论在不动点理论、算子谱理论、 泛函分析、常 (偏) 微分方程和积分方程等领域具有广泛的应用.

球拓扑的有关内容将会在第 4 节中结合其球覆盖性质的刻画进行介绍.

本文将简要介绍 Banach 空间中球覆盖性质 ${ }^{[12]}$ (在文献 [13] 中也被称为 spheres covering by balls) 的研究进展. 本文分为 6 节, 第 1 节为背景介绍; 第 2 节介绍有限维空间的球覆盖问题, 主要讨论 有关最小球覆盖的个数和半径问题; 第 3 节介绍可分性与球覆盖性质之间的联系; 第 4 节介绍利用 球覆盖性质刻画的 Banach 空间几何和拓扑性质; 第 5 节是球覆盖性质的稳定性的有关内容; 第 6 节给出尚待解决的问题.

文中 $X$ 表示 Banach 空间, $X^{*}$ 则表示 $X$ 的对偶空间. 用 $B_{X}$ 来表示 Banach 空间 $X$ 中的闭单 位球, $S_{X}$ 则表示 Banach 空间 $X$ 中的单位球面. $B(x, r)$ (相应地, $\bar{B}(x, r)$ ) 为中心在点 $x$ 、半径为 $r$ 的 开 (相应地, 闭) 球. 对于 Banach 空间 $X$, 其单位球面 $S_{X}$ 可以被 $X$ 中一族不包含原点的开 (相应地, 闭) 球族 $\mathcal{B}$ 所覆盖. 特别当 $X$ 为可分空间 (相应地, 有限维空间) 时, 球族 $\mathcal{B}$ 可以选取由可数多个 (相 应地, 有限多个) 半径任意小的球所构成. 这就自然产生了如下的问题:

问题 1.1 哪些不可分 Banach 空间的球面可以被可数个不包含原点的球构成的球族所覆盖?

如果 Banach 空间 $X$ 的单位球面可以被可数个不包含原点的球构成的球族所覆盖, 那么就称 $X$ 具有球覆盖性质. 因为一方面不包含原点的开球 $B$ 可以表示成一列含于 $B$ 的闭球的并; 而另一方面, 任意不包含原点的闭球都可以含于一个不包含原点的开球, 所以, 在无穷维 Banach 空间 $X$ 中, 考虑 球覆盖问题时, 只需要去研究球面的开球覆盖或者闭球覆盖其中一种情形即可.

以下举例说明存在具有球覆盖性质的不可分 Banach 空间:

例 1.1 假设 $X=\ell_{\infty}$ 且对于 $n=1,2, \ldots, e_{n}=\chi_{\{n\}} \in \ell_{\infty}$; 对任意 $0<\delta<1$ 和 $n \in \mathbb{N}$, 可以假 设 $x_{n}=(1+\delta) e_{n}$, 则 $\mathcal{B}=\left\{\bar{B}\left( \pm x_{n}, 1\right): n \in \mathbb{N}\right\}$ 就是 $S_{\ell_{\infty}}$ 的一个可数球覆盖.

对于有限维空间单位球面的球覆盖, 有下面的问题:

问题 1.2 设 $\mathfrak{B}(X)$ 为 $n$ 维空间 $X$ 中单位球面的所有球覆盖 $\mathcal{B}$ 所构成的集族, $\mathcal{B}^{\sharp}$ 表示 $\mathcal{B}$ 的基

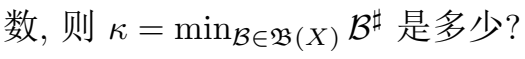

这里先介绍在研究 Banach 空间球覆盖性质中起到重要作用的次微分概念及其相关结果, 对于 
Banach 空间 $X$ 上的凸函数 $f$, 其次微分映射 $\partial f: X \rightarrow 2^{X^{*}}$ 定义如下:

$$
\partial f(x)=\left\{x^{*} \in X^{*}: f(y)-f(x) \geqslant\left\langle x^{*}, y-x\right\rangle, \forall y \in X\right\}, \quad \forall x \in X .
$$

注意到, 如果 $f$ 连续, 则对于任意 $x \in X, \partial f(x)$ 为非空 $w^{*}$ 紧凸集 (参见文献 [14]). 若对于任意 $x \in X$ 都有 $\varphi(x) \in \partial f(x)$, 则称单值映射 $\varphi: X \rightarrow X^{*}$ 为集值映射 $\partial f: X \rightarrow 2^{X^{*}}$ 的一个选择. 而连续 凸函数 $f: X \rightarrow \mathbb{R}$ 在 $x$ 处 Gâteaux 可微当且仅当 $\partial f(x)$ 是单点集. 所以, 此时有 $\partial f(x)=\{d f(x)\}$, 这 里 $d f(x) \in X^{*}$ 表示 $f$ 在 $x$ 处的 Gâteaux 导数.

接下来的这个定理在球覆盖性质的研究中起到了非常关键的作用.

定理 $1.1^{[15]}$ 设 $X$ 为 Banach 空间, $\left\{z_{n}\right\} \subset S_{X}$, 则满足对于任意 $n \in \mathbb{N}$, 有 $x_{n} \in \mathbb{R}^{+} z_{n}$, 且 $\left\|x_{n}\right\| \geqslant r_{n}$ 的序列 $\mathcal{B} \equiv\left\{B\left(x_{n}, r_{n}\right)\right\}$ 构成 $X$ 的球覆盖当且仅当对于范数 $\|\cdot\|$ 的次微分映射 $\partial\|\cdot\|$ 的任 意选择 $\varphi$, 都有 $\left\{\varphi\left(x_{n}\right)\right\}$ 正分离 $X$ 中的点, 即对于 $X$ 中的任意非零点 $x$, 都满足 $\sup _{n \in \mathbb{N}}\left\langle\varphi\left(x_{n}\right), x\right\rangle>0$.

证明 充分性. 不失一般性, 对于任意 $n \in \mathbb{N}$, 假设 $\left\|z_{n}\right\|=1$ 且 $r_{n}=\left\|x_{n}\right\|$. 取定次微分映射 $\partial\|\cdot\|$ 的一个选择 $\psi$. 如果结论不真, 则存在 $y \in S_{X}$ 使得对于任意 $k, n \in \mathbb{N}$, 有 $y \notin B\left(k z_{n}, k\right)$, 即

$$
\left\|k z_{n}-y\right\| \geqslant k, \quad \forall k, n \in \mathbb{N} .
$$

也等价于,

$$
\frac{\left\|z_{n}-\frac{1}{k} y\right\|-\left\|z_{n}\right\|}{\frac{1}{k}} \geqslant 0, \quad \forall k, n \in \mathbb{N} .
$$

注意到 $\|\psi(x)\|=1$ 且对于 $X$ 中的任意非零点 $x$, 都有 $\langle\psi(x), x\rangle=\|x\|$, 那么由 (1.3) 可得

$$
\left\langle\psi\left(z_{n}-\frac{1}{k} y\right),-y\right\rangle \geqslant \frac{\left\|z_{n}-\frac{1}{k} y\right\|-\left\|z_{n}\right\|}{\frac{1}{k}} \geqslant 0, \quad \forall k, n \in \mathbb{N} .
$$

所以,

$$
\left\langle\psi\left(z_{n}-\frac{1}{k} y\right), y\right\rangle \leqslant 0, \quad \forall k, n \in \mathbb{N} .
$$

对于任意给定的 $n \in \mathbb{N}$, 设 $z_{n}^{*}$ 为集合 $\left(\psi\left(z_{n}-\frac{1}{k} y\right)\right)_{k=1}^{\infty}$ 的 $w^{*}$ 聚点, 则都有 $z_{n}^{*} \in \partial\left\|z_{n}\right\|$ 且 $\left\langle z_{n}^{*}, y\right\rangle \leqslant 0$. 现在定义 $\partial\|\cdot\|$ 的新的选择如下:

$$
\varphi(x)= \begin{cases}\psi(x), & x \neq z_{n}, \\ z_{n}^{*}, & x=z_{n} .\end{cases}
$$

而此时, $\left\{\varphi\left(z_{n}\right)\right\}$ 不能正分离 $X$ 中的点.

必要性. 设 $\mathcal{B}=\left\{B\left(x_{n}, r_{n}\right)\right\}$ 为 $S_{X}$ 的球覆盖, 则对于任意 $y \in S_{X}$, 存在 $m \in \mathbb{N}$ 使得 $y \in B\left(x_{m}\right.$, $\left.\left\|x_{m}\right\|\right)$. 于是对于某一正数 $\delta>0$ 和任意 $0<t \leqslant 1$, 有

$$
\frac{\left\|x_{m}-t y\right\|-\left\|x_{m}\right\|}{t} \leqslant\left\|x_{m}-y\right\|-\left\|x_{m}\right\| \equiv-\delta .
$$

因为

$$
\begin{aligned}
& \max _{x^{*} \in \partial\left\|x_{m}\right\|}\left\langle x^{*},-y\right\rangle=\lim _{t \rightarrow 0^{+}} \frac{\left\|x_{m}-t y\right\|-\left\|x_{m}\right\|}{t} \leqslant-\delta, \\
& \min _{x^{*} \in \partial\left\|x_{m}\right\|}\left\langle x^{*}, y\right\rangle \geqslant \delta,
\end{aligned}
$$

所以, (1.7) 等价于对于次微分映射 $\partial\|\cdot\|$ 的任意选择 $\varphi$, 都有 $\left\langle\varphi\left(x_{m}\right), y\right\rangle \geqslant \delta>0$. 
下面给出例子来作为定理 1.1 的应用.

例 1.2 设 $X$ 为 $n$ 维 Euclid 空间 $\mathbb{R}^{n}$, 则单位球面 $S_{X}$ 的球覆盖中包含球个数的最小值 $\kappa$ 为 $n+1$.

证明 注意到 Euclid 范数 $\|\cdot\|$ 在 $X \backslash\{0\}$ 中处处可微, 且对于任意 $x \neq 0$ 有 $d\|x\|=x /\|x\|$. 先 证明 $\kappa \geqslant n+1$. 假设 $\mathcal{B}=\left\{B\left(x_{j}, r_{j}\right)\right\}_{j=1}^{m}$ 为 $S_{X}$ 的最小球覆盖, 此时有 $\kappa=m$, 则由定理 1.1 可知, $\left\{\varphi_{j}\right\}_{j=1}^{m}$ 正分离 $X$ 中的点, 而这里对于所有 $1 \leqslant j \leqslant m$ 都有 $\varphi_{j} \equiv d\left\|x_{j}\right\|=x_{j} /\left\|x_{j}\right\|$. 因此, $m \geqslant n+1$, 即 $\kappa \geqslant n+1$.

另一方面, 假设 $\left(e_{j}\right)_{j=1}^{n}$ 为 $X$ 的标准向量基,

$$
e_{0}=-\frac{1}{\sqrt{n}} \sum_{j=1}^{n} e_{j},
$$

则 $\left\{e_{j}\right\}_{j=0}^{n}$ 正分离了 $X$ 中的点. 由定理 1.1 可得 $S_{X}$ 具有 $n+1$ 个球构成的球覆盖. 所以, $\kappa \leqslant n+1$. 综上, $\kappa=n+1$.

\section{2 有限维空间的最小球覆盖问题}

在 Banach 空间 $X$ 中, 用 $\mathscr{B}(X)$ 来表示所有单位球面 $S_{X}$ 的所有球覆盖 $\mathcal{B}$ 所构成的集族. 如果 $\mathcal{B}_{0} \in \mathscr{B}(X)$ 满足 $\mathcal{B}_{0}^{\sharp}=\min \left\{\mathcal{B}^{\sharp}: \mathcal{B} \in \mathscr{B}(X)\right\}$, 即 $\mathcal{B}_{0}$ 的基数 $\mathcal{B}_{0}^{\sharp}$ 在 $\left\{\mathcal{B}^{\sharp}: \mathcal{B} \in \mathscr{B}(X)\right\}$ 中取到最小值, 那 么就称 $\mathcal{B}_{0}$ 为最小球覆盖. 我们也用 $\mathcal{B}_{\text {min }}$ 来表示 $S_{X}$ 的最小球覆盖, $\kappa \equiv \kappa(X)=\mathcal{B}_{\min }^{\sharp}$ 表示 $X$ 的所有 球覆盖基数中的最小值.

\section{$2.1 n$ 维空间球覆盖的最小基数}

对于 $n(n>1)$ 维 $\ell_{p}$ 空间, 有下面的定理:

定理 2.1 设 $X=\ell_{p}^{n}$, 则

(i) (参见文献 [12]) 当 $1<p<\infty$ 时, $\kappa=n+1$;

(ii) 当 $p=1$ 时, 若 $n=2$, 则有 $\kappa=4$; 若 $n>2$, 则有 $\kappa=n+1^{1)}$;

(iii) (参见文献 [12]) 当 $p=\infty$ 时, $\kappa=2 n$.

注 2.1 $\mathrm{Hu}$ 和 Zhao ${ }^{1)}$ 关于定理 2.1(ii) 的研究思路是, 因为 $\ell_{1}^{n}$ 的对偶空间单位球 $B_{\ell_{\infty}^{n}}$ 的暴露点 集就是 $\sum_{j=1}^{n} \pm e_{j}$, 所以, 根据定理 1.1 , 只需证明当 $n>2$ 时, 最少可以取集合 $\left\{\sum_{j=1}^{n} \pm e_{j}\right\}$ 中的 $n+1$ 个元素就能够分离 $\ell_{1}^{n}$ 中的点即可.

定理 $2.2^{[12]}$ 设 $X$ 是 $n$ 维空间, 则

(i) $n+1 \leqslant \kappa \leqslant 2 n$;

(ii) 当 $X$ 光滑时, $\kappa=n+1$;

(iii) $\kappa=2 n$ 当且仅当 $X$ 等距同构于 $\ell_{\infty}^{n}$.

定理 $2.3^{[16]}$ 设 $n, k \in \mathbb{N}$ 且 $n+1 \leqslant k \leqslant 2 n$, 则存在 $n$ 维空间 $X$ 使得 $\kappa(X)=k$.

1) Hu Z, Zhao X. On minimal ball-coverings of $\ell_{1}^{n}$. Preprint, 2017 


\section{2 最小球覆盖的最优半径}

对于 $S_{X}$ 的球覆盖 $\mathcal{B}=\left\{B\left(x_{i}, r_{i}\right)\right\}_{i \in I}$, 称 $r_{\mathcal{B}} \equiv \sup _{i \in I} r_{i}$ 为 $\mathcal{B}$ 的半径. 用 $\gamma_{\mathrm{inf}}$ 表示 $\left\{r_{\mathcal{B}}: \mathcal{B}\right.$ 为最小 球覆盖 $\}$ 的下确界. 在介绍有关结果之前, 先介绍一个引理.

引理 $2.1{ }^{[17]}$ 设 $\Omega$ 为 Euclid 空间 $\mathbb{R}^{n}(n \geqslant 2)$ 中的 $n$ - 单形, 顶点集合为 $\left\{x_{i}\right\}_{i=0}^{n}$ 且 $0 \in \operatorname{int}(\Omega)$. 设 $S$ 为 $\Omega$ 的外接超球面, $r$ 为 $S$ 的半径且 $\rho_{i j}=\left\|x_{i}-x_{j}\right\|$. 类似地, 设 $\Omega_{j}$ 为 $n$ - 单形 $\operatorname{co}\left[\left\{x_{i}\right\}_{i \neq j} \cup\{0\}\right]$, $S_{j}$ 为 $\Omega_{j}$ 的外接超球面, $r_{j}$ 为 $S_{j}$ 的半径且 $d_{j}$ 为 0 到 $M_{j}=\operatorname{co}\left(x_{i}\right)_{i \neq j}$ 的距离, 则

$$
r^{2}=-\frac{1}{2} \frac{D_{0}\left(x_{0}, x_{1}, \ldots, x_{n}\right)}{D\left(x_{0}, x_{1}, \ldots, x_{n}\right)}
$$

其中

$$
D_{0} \equiv D_{0}\left(x_{0}, x_{1}, \ldots, x_{n}\right)=\left|\begin{array}{ccccc}
0 & \rho_{01}^{2} & \rho_{02}^{2} & \cdots & \rho_{0 n}^{2} \\
\rho_{10}^{2} & 0 & \rho_{12}^{2} & \cdots & \rho_{1 n}^{2} \\
\vdots & \vdots & \vdots & \ddots & \vdots \\
\rho_{n 0}^{2} & \rho_{n 1}^{2} & \rho_{n 2}^{2} & \cdots & 0
\end{array}\right|,
$$

且

$$
D \equiv D\left(x_{0}, x_{1}, \ldots, x_{n}\right)=\left|\begin{array}{ccccc}
0 & 1 & 1 & \cdots & 1 \\
1 & 0 & \rho_{01}^{2} & \cdots & \rho_{0 n}^{2} \\
\vdots & \vdots & \vdots & \ddots & \vdots \\
1 & \rho_{n 0}^{2} & \rho_{n 1}^{2} & \cdots & 0
\end{array}\right|,
$$

$r_{j}=\frac{r^{2}}{2 d_{j}}(j=0,1, \ldots, n), r^{n+1} \leqslant\left(\frac{2}{n}\right)^{n+1} \prod_{j=0}^{n} r_{j}$. 特别地, 当 $\Omega$ 为正则 $n$ - 单形时, $\rho \equiv \rho_{i j}=$ $\sqrt{\frac{2(n+1)}{n}} r(0 \leqslant i, j \leqslant n)$.

定理 2.4 [18] 设 $X$ 为满足 $n \geqslant 2$ 的 $n$ 维 Euclid 空间, $\left\{x_{i}\right\}_{i=0}^{n}$ 为 $\frac{n}{2} S_{X}$ 的内接正则 $n$ - 单形的顶 点集, 则 $S_{X} \subset \bigcup_{i=0}^{n} \bar{B}\left(x_{i}, \frac{n}{2}\right)$.

证明 设 $y_{i}=\frac{2}{n}\left\{x_{i}\right\}_{i=0}^{n}$, 则 $\left\{y_{i}\right\}_{i=0}^{n}$ 为 $S_{X}$ 内接正则 $n$ - 单形 $\Omega$ 的顶点集. 由引理 2.1 可得

$$
\rho=\rho_{i j}=\left\|y_{i}-y_{j}\right\|=\sqrt{\frac{2(n+1)}{n}}, \quad 0 \leqslant i \neq j \leqslant n .
$$

因为任意 $\Omega_{i}=\operatorname{co}\left[\left\{y_{i}\right\}_{i \neq j} \cup\{0\}\right]$ 的外接超球面是球 $B\left(r z_{i}, r\right)$ 的球面, 其中 $z_{i} \in S_{X}$,

$$
r^{2}=-\frac{1}{2} \frac{D_{0}\left(y_{0}, y_{1}, \ldots, y_{n}\right)}{D\left(y_{0}, y_{1}, \ldots, y_{n}\right)}
$$

这里

$$
D_{0} \equiv D_{0}\left(0, y_{1}, \ldots, y_{n}\right)=-n\left(-\rho^{2}\right)^{n-1}
$$

且

$$
D \equiv D\left(0, y_{1}, \ldots, y_{n}\right)=\left(-\rho^{2}\right)^{n-1}\left[2 n-(n-1) \rho^{2}\right],
$$

于是, 有 $r=\sqrt{\frac{-D_{0}}{2 D}}=\frac{n}{2}$. 设 $M_{j}=\operatorname{co}\left(y_{i}\right)_{i \neq j}$ 且 $d_{j}=\operatorname{dist}\left(0, M_{j}\right)$, 则 $r=\frac{1}{2 d_{j}}, d_{j}=\frac{1}{n}$. 
现在考虑内接正则 $n$ - 单形 $\Omega^{\prime} \equiv \operatorname{co}\left(z_{i}\right)_{i=0}^{n}$, 相应地, 有 $M_{j}^{\prime}=\operatorname{co}\left(z_{i}\right)_{i \neq j}, \Omega_{j}^{\prime}=\operatorname{co}\left(z_{i}\right)_{i \neq j} \cup(0)$ 且 $d_{j}^{\prime}=\operatorname{dist}\left(0, M_{j}\right)\left(=d=\frac{1}{n}\right), 0 \leqslant i \leqslant n$.

下证 $S_{X} \subset \bigcup_{i=0}^{n} \bar{B}\left(\frac{n}{2} z_{i}, \frac{n}{2}\right)$. 否则, 存在 $x \in S_{X} \backslash \bigcup_{i=0}^{n} \bar{B}\left(\frac{n}{2} z_{i}, \frac{n}{2}\right)$, 则对于任意 $0 \leqslant i \leqslant n$ 都有 $\left\|x-\frac{1}{n} z_{i}\right\|>\frac{1}{n}$. 这说明 $\left\langle x, z_{i}\right\rangle<\frac{1}{n}, i=0,1, \ldots, n$. 于是,

$$
\frac{1}{n} \leqslant \max _{y \in \Omega^{\prime}}\langle x, y\rangle=\max _{y \in \operatorname{co}\left(z_{i}\right)_{i=0}^{n}}\langle x, y\rangle=\max _{0 \leqslant i \leqslant n}\left\langle x, z_{i}\right\rangle<\frac{1}{n},
$$

矛盾. 所以, $S_{X} \subset \bigcup_{i=0}^{n} \bar{B}\left(\frac{n}{2} z_{i}, \frac{n}{2}\right)$. 因为 $\Omega$ 等距同构于 $\Omega^{\prime}$, 所以, $S_{X} \subset \bigcup_{i=0}^{n} \bar{B}\left(x_{i}, \frac{n}{2}\right)$.

推论 2.1 设 $X$ 为满足 $n \geqslant 2$ 的 $n$ 维 Euclid 空间, 则 $\gamma_{\mathrm{inf}} \leqslant \frac{n}{2}$.

2008 年, 林国琛和沈喜生 ${ }^{[19]}$ 利用神经网络方法研究了 $n$ 维 Euclid 空间中基数为 $\mathcal{B}^{\sharp}=m$ 的球 覆盖 $\mathcal{B}$ 的最小半径问题. 他们给出了最小半径 $\gamma_{\mathrm{inf}}$ 的计算公式. 部分具体结果如下:

引理 $2.2^{[20]}$ 设 $\Omega=\operatorname{co}\left\{x_{i}\right\}_{i=1}^{n+1}$ 为 $n$ 维 Euclid 空间中的 $n$ - 单形, $R$ 为其外接超球面的半径, $P \in \operatorname{int} \Omega\left(\Omega\right.$ 的内部) 且 $d_{i}$ 为 $P$ 到 $\Omega_{i}=\operatorname{co}\left\{x_{j}: 1 \leqslant j \leqslant n+1, j \neq i\right\}$ 的距离, 则 $\prod_{i=1}^{n+1} d_{i} \leqslant\left(\frac{R}{n}\right)^{n+1}$, 并且等号成立当且仅当 $\Omega$ 为正则单形.

定理 $2.5^{[19]}$ 设 $X$ 为满足 $n \geqslant 2$ 的 $n$ 维 Euclid 空间, 则 $\gamma_{\mathrm{inf}}=\frac{n}{2}$.

证明 只需证明 (i) 对于任意满足 $0 \in \operatorname{int} \operatorname{co}\left\{x_{i}\right\}_{i=1}^{n+1}$ 的序列 $\left\{x_{i}\right\}_{i=1}^{n+1} \subset S_{\mathbb{R}^{n}}$, 有

$$
\max _{\left\{x_{i}\right\}_{i=1}^{n+1} \subset S_{\mathbb{R}}^{n}} \min _{\|x\|=1} \max _{1 \leqslant i \leqslant n+1}\left\langle x_{i}, x\right\rangle \leqslant \frac{1}{n} ;
$$

(ii) 上式取到最大值当且仅当 $\operatorname{co}\left\{x_{i}\right\}_{i=1}^{n+1}$ 为正则单形.

设 $\Omega=\operatorname{co}\left\{x_{i}\right\}_{i=1}^{n+1}$ 且 $d_{i}=d\left(0, \Omega_{i}\right)$, 则由引理 2.2 , 有 $d_{i_{0}} \equiv \min _{1 \leqslant i \leqslant n+1} d_{i} \leqslant \frac{1}{n}$. 再设 $w$ 为 0 在 $\Omega_{i_{0}}$ 中的最佳逼近点且 $x=\frac{w}{\|w\|}$, 则有 $\left\langle x_{i_{0}}, w\right\rangle \leqslant 0$ 且 $\max _{1 \leqslant i \leqslant n+1}\left\langle x_{i}, x\right\rangle=d_{i_{0}} \leqslant \frac{1}{n}$. 于是当 $\Omega$ 非正则时, $d_{i_{0}}<\frac{1}{n}$; 而当 $\Omega$ 正则时, $d_{i_{0}}=\frac{1}{n}$. 选择 $\left\{B\left(\frac{n}{2} x_{i}, \frac{n}{2}\right)\right\}_{i=1}^{n+1}$ 即可得 $\gamma_{\mathrm{inf}}=\frac{n}{2}$.

注 2.2 最近, 陈泽宇经过讨论进一步得到对 $n$ 维 Euclid 空间 $\mathbb{R}^{n}$, 下确界 $\gamma_{\mathrm{inf}}$ 不可达.

注 2.3 由定理 2.1(iii) 和例 1.1 可得, 当 $n>2$ 时, $\gamma_{\mathrm{inf}}\left(\ell_{\infty}^{n}\right)=1$. 但是除此结果和对 Euclid 空间 的讨论之外, 对一般有限维空间 $X$ 的 $\gamma_{\mathrm{inf}}$ 还没得到相应结果. 甚至不知道当 $1 \leqslant p \neq 2<\infty$ 时, $\ell_{p}^{n}$ 的 $\gamma_{\text {inf }}$ 是多少.

这就自然产生了如下问题:

问题 2.1 对于 $X=\ell_{p}^{n}, \gamma_{\mathrm{inf}}=$ ?

注 2.4 对于有限维赋范空间, 文献 [21-24] 也取得了许多有趣的研究成果. 例如, 林丽华等 [23] 证明了对于 $n$ 维赋范空间, 如果其最小球覆盖基数为 $2 n-1$, 则它一定包含一个与 $\left(\mathbb{R}^{n-1},\|\cdot\|_{\infty}\right)$ 等距 同构的 $n-1$ 维子空间.

称 $S_{X}$ 的球覆盖 $\mathcal{B}$ 为对称的, 如果 $B \in \mathcal{B}$ 可以推出 $-B \in \mathcal{B}$. 关于对称球覆盖, 傅瑞瑜和程立 新 [21] 得到了对于 $n$ 维赋范空间 $X$, 对称的最小球覆盖的基数为 $2 n$. 他们还证明了 $n$ 维 Euclid 空间 $X=\mathbb{R}^{n}$, 最小对称球覆盖的半径的下确界为 $\frac{\sqrt{n}}{2}$, 并且其下确界不可达. 文献 [25] 还讨论了关于遗传 不可分解空间的球覆盖性质.

\section{3 球覆盖性质与可分性}

我们知道 Banach 空间 $X$ 可分当且仅当对于任意的 $\varepsilon>0$, 存在由可数个球构成的球覆盖 $\mathcal{B}$ 满足 半径 $r(\mathcal{B})<\varepsilon$. 所以, 可分空间都具有球覆盖性质. 如果 $X$ 不可分, 则对于任意 $\varepsilon>0$, 存在不可数的 
网 $\left\{x_{\iota}\right\} \subset S_{X}$, 使得

$$
\left\|x_{\xi}-x_{\eta}\right\|>1-\varepsilon, \quad \forall \xi \neq \eta .
$$

由此可知, 如果 $X$ 具有满足 $r(\mathcal{B})<1 / 2$ 的可数球覆盖 $\mathcal{B}$, 则 $X$ 一定可分.

另一方面, 根据凸集分离定理, 对于具有球覆盖性质的 Banach 空间 $X$, 其对偶空间 $X^{*}$ 一定 $w^{*}$ 可分. 因为对于自反的 Banach 空间 $X$, 它具有球覆盖性质当且仅当其可分.

本节将主要关注如下两个问题:

问题 3.1 是否存在实数 $r_{0}>0$ 满足

(i) 当 Banach 空间 $X$ 具有满足 $r(\mathcal{B})<r_{0}$ 的可数球覆盖 $\mathcal{B}$ 时, $X$ 一定可分;

(ii) 当 $r>r_{0}$ 时, 存在不可分的 Banach 空间 $X$ 具有满足 $r(\mathcal{B})<r$ 的可数球覆盖 $\mathcal{B}$.

问题 3.2 如果 $X^{*}$ 是 $w^{*}$ - 可分的, $X$ 是否可赋予等价范数, 使其具有球覆盖性质?

对于球族 $\mathcal{B}$, 如果 $\mathcal{B}$ 中每个球的半径都小于等于 $r$, 则将其记为 $\mathcal{B}(r)$. 结合例 1.1 , 下述定理得到 了 $r_{0}=1$, 从而肯定地回答了问题 3.1 .

定理 $3.1^{[12]}$ 设 $0<r<1$, 如果 $X$ 具有可数球覆盖 $\mathcal{B}$ 满足 $r(\mathcal{B}) \leqslant r$, 则 $X$ 可分.

证明 设 $\mathcal{B}=\left\{B\left(x_{n}, r_{n}\right)\right\}_{n=1}^{\infty}$ 为 $S_{X}$ 的可数球覆盖, 满足对于任意 $n \in \mathbb{N}$ 有 $0<r_{n} \leqslant r$. 再设 $\eta=\frac{1-r}{2}$ 且 $r_{\eta}=r+\eta$, 则 $\mathcal{B}_{1}\left(r_{\eta}\right) \equiv\left\{B\left(x_{n}, r_{n}+\eta\right)\right\}_{n=1}^{\infty}$ 覆盖了 $B_{X} \backslash(1-\eta) B_{X}$. 于是, 存在由可数个球 构成的集族 $\mathcal{B}\left(r_{\eta}\right)$, 其并覆盖了 $B_{X}$. 因为 $\mathcal{B}\left(r_{\eta}\right)$ 中的每个球又可以被一列半径小于等于 $r_{\eta}^{2}$ 的球所覆 盖, 所以, $B_{X}$ 可以被由可数个半径小于等于 $r_{\eta}^{2}$ 的球所构成的集族 $\mathcal{B}_{2}\left(r_{\eta}^{2}\right)$ 所覆盖. 如此下去, 对于任 意的 $n \in \mathbb{N}$, 可以找到对应的由可数个半径小于等于 $r_{\eta}^{n}$ 的集族 $\mathcal{B}_{n}\left(r_{\eta}^{n}\right)$. 因为当 $n \rightarrow \infty$ 时, $r_{\eta}^{n} \rightarrow 0$, 所 以, $B_{X}$ 被一列半径任意小的球所覆盖, 这就说明了 $X$ 可分.

定理 3.2 假设 $X$ 是 (Gâteaux) 光滑 Banach 空间, 则 $X$ 具有球覆盖性质当且仅当 $X^{*}$ 是 $w^{*}$ 可分的.

证明 只需证明充分性. 因为 $X$ 为 Gâteaux 光滑的, 所以, 任意范数可达泛函 $x^{*} \in S_{X^{*}}$ 都 是 $B_{X^{*}}$ 的 $w^{*}$ - 暴露点, 即存在 $x \in S_{X}$ 使得 $d\|x\|=x^{*}$ (参见文献 [14]). 根据 Bishop-Phelps 定理 ${ }^{[26]}$, 范数可达泛函在 $X^{*}$ 中稠密. 此时由 $X^{*}$ 是 $w^{*}$ - 可分的知, 存在一列 $\left\{z_{n}^{*}\right\} \subset S_{X^{*}}$ 正分离 $X$ 中 的点. 于是存在 $B_{X^{*}}$ 的一列 $w^{*}$ - 暴露点 $\left\{y_{n}^{*}\right\}$ 正分离 $X$ 中的点. 再设 $\left\{y_{n}\right\} \subset S_{X}$, 使得对于任意 $n \in \mathbb{N}$ 都有 $d\left\|y_{n}\right\|=y_{n}^{*}$. 根据 1.1 可得, 存在可数球覆盖 $\mathcal{B}=\left\{B\left(x_{n}, r_{n}\right)\right\}$, 满足对于任意 $n \in \mathbb{N}$ 都有 $x_{n} \in \mathbb{R}^{+} y_{n}$.

问题 3.2 的答案同样也是肯定的. 文献 [15] 采用了关于双正交系的广义 Pelczyński 引理得到了下 述结果:

定理 $3.3^{[15]}$ 设 $X$ 是对偶 $w^{*}$ - 可分的 Banach 空间, 则对于任意 $\varepsilon>0$, 存在 $X$ 的等价范数 $|\cdot|$, 使得

(i) $(1+\varepsilon)^{-1}\|x\| \leqslant|x| \leqslant(1+\varepsilon)\|x\|, \forall x \in X$;

(ii) $X$ 关于 $|\cdot|$ 具有球覆盖性质.

2009 年, Fonf 和 Zanco ${ }^{[13]}$ 也独立证明了对偶 $w^{*}$ 可分的 Banach 空间 $X$ 可以赋予等价范数, 使 得 $X$ 具有由可数个一致有界的球所构成的球覆盖.

定理 $3.4^{[13]}$ 对于 Banach 空间, 下列等价:

(i) $X^{*}$ 是 $w^{*}$ - 可分的;

(ii) 对于任意 $\varepsilon>0$, 存在 $X$ 的 $(1+\varepsilon)$ - 等价范数和 $R>0$, 使得在此范数下, $X$ 具有满足 $r(\mathcal{B}) \leqslant R$ 的球覆盖 $\mathcal{B}$. 
Cheng 等 ${ }^{[27]}$ 进一步给出了上述 Fonf 和 Zanco 再赋范定理的精确量化版本.

定理 $3.5^{[27]}$ 设对偶 $w^{*}$ 可分的 Banach 空间, 对于任意 $0<\varepsilon<\frac{1}{3}$, 存在满足 $\|\cdot\| \leqslant|\cdot| \leqslant(1+\varepsilon)\|\cdot\|$ 的等价范数 $|\cdot|$, 使得 $(X,|\cdot|)$ 有半径 $r(\varepsilon) \leqslant \frac{1+\varepsilon}{\varepsilon}$ 的球覆盖 $\mathcal{B}(r(\varepsilon))$.

文献 [27] 还证明了对充分小的 $0<\varepsilon<\frac{1}{3}$, Banach 空间 $L[0,1]$ 可以赋 $(1+\varepsilon)-$ 等价范数 $|\cdot|$, 使得 关于此等价范数, $L[0,1]$ 有 $r(\varepsilon)$ 任意接近 $\frac{1}{\varepsilon}$ 的球覆盖, 即当 $\varepsilon \rightarrow 0$ 时, $\varepsilon r(\varepsilon) \rightarrow 1$. 但是 $\frac{1}{\varepsilon}$ 不可达.

最近, Guirao 等 [28] 研究了空间的可分性与其对偶空间上的球覆盖性质之间的关系, 并且利用空 间再赋范后其对偶空间的球覆盖性质刻画了空间的可分性.

\section{4 利用球覆盖性质来刻画的几何性质和拓扑性质}

本节主要介绍如何利用有限维子空间的球覆盖性质来刻画整个空间的几何性质和拓扑性质.

先从超自反空间的刻画开始, 对于 Banach 空间 $X$ 的球覆盖 $\mathcal{B}$, 如果存在 $\alpha>0$ 使得任意 $B \in \mathcal{B}$ 都有 $\inf _{b \in B}\|b\| \geqslant \alpha$, 则称 $\mathcal{B}$ 为 $\alpha$ - 远离原点. 称 Banach 空间 $Y$ 在 Banach 空间 $X$ 中有限表示, 如果 满足对任意 $\varepsilon>0$ 和任意有限维子空间 $Y_{0} \subset Y$, 存在 $X$ 的子空间 $X_{0}$ 和线性同胚 $T: Y_{0} \rightarrow X_{0}$ 使得 $\|T\|\left\|T^{-1}\right\|<1+\varepsilon$. 称 $X$ 超自反, 如果任意在 $X$ 中有限表示的 Banach 空间 $Y$ 均自反.

定理 4.1 [16] 设 $X$ 为 Banach 空间, 则 $X$ 超自反当且仅当存在 $X$ 的等价范数使得在新范数下 有正值函数 $f, g: \mathbb{N} \mapsto \mathbb{R}^{+}$满足对任意 $n \in \mathbb{N}$ 和任意 $n$ 维子空间 $Y$, 都有 $Y$ 的最小球覆盖 $\mathcal{B}$ 满足

(i) $\mathcal{B}^{\#}=n+1$;

(ii) $r(\mathcal{B}) \leqslant f(n)$;

(iii) $\mathcal{B}$ 为 $g(n)$ - 远离原点.

接下来是范数稠 Gâteaux 可微的 Banach 空间的刻画:

定理 4.2 设 $X$ 为范数稠 Gâteaux 可微的 Banach 空间, 则 $X$ 具有球覆盖性质当且仅当存在一 列 $B_{X^{*}}$ 中的 $w^{*}$ - 暴露点 $\left\{x_{n}^{*}\right\}$ 正分离 $X$ 中的点.

证明 充分性. 注意到对于任意 $x_{m}^{*} \in\left\{x_{n}^{*}\right\}$, 存在 $x_{m} \in S_{X}$ 使得 $d\left\|x_{m}\right\|=x_{m}^{*}$, 而 $\partial\left\|x_{m}\right\|=$ $\left\{d\left\|x_{m}\right\|\right\}$. 利用定理 1.1 即完成所证.

必要性. 设 $\mathcal{B}=\left\{B\left(x_{n}, r_{n}\right)\right\}_{n=1}^{\infty}$ 为 $S_{X}$ 的球覆盖, 满足对于任意 $n \in \mathbb{N}$ 都有 $\left\|x_{n}\right\| \geqslant r_{n}$. 因为 $X$ 的光滑点 (即范数的 Gâteaux 可微点) 在 $X$ 中稠密, 所以, 对任意 $x_{n}$, 存在一列 $\left(x_{n j}\right) \subset X$ 使得 $x_{n j} \rightarrow x_{n}$. 显然, $B\left(x_{n}, r_{n}\right) \subset \bigcup_{j=1}^{\infty} B\left(x_{n j},\left\|x_{n j}\right\|\right)$. 由此可得 $\mathcal{B}_{1} \equiv\left\{B\left(x_{i j},\left\|x_{i j}\right\|\right)\right\}_{i, j \in \mathbb{N}}$ 也为 $S_{X}$ 的球覆 盖, 且 $\mathcal{B}_{1}$ 中的每个球都以 $X$ 中的光滑点为球心. 再设 $\varphi_{i j}=d\left\|x_{i j}\right\|$. 结合定理 1.1 可知, $\left\{\varphi_{i j}\right\}_{i j, \in \mathbb{N}}$ 正 分离了 $X$ 中的点.

在此先回顾有关定义, 称 Banach 空间 $X$ 为 Gâteaux 可微空间, 若任意定义在 $X$ 上的连续凸函 数均稠 Gâteaux 可微. 可分 Banach 空间和 Gâteaux 光滑的 Banach 空间都是 Gâteaux 可微空间 (参 见文献 [14]).

关于 Gâteaux 可微空间, 有如下推论:

推论 4.1 设 $X$ 为 Gâteaux 可微空间, 则 $X$ 具有球覆盖性质当且仅当存在 $B_{X *}$ 中的 $w^{*}$ - 暴露 点列 $\left\{x_{n}^{*}\right\}$ 正分离 $X$ 中的点.

称 Banach 空间 $X$ 为 (Gâteaux) 光滑的, 如果对于任意 $x(\neq 0) \in X$, 存在 $x^{*} \in X^{*}$ 使得

$$
\lim _{t \rightarrow 0} \frac{\|x+t y\|-\|x\|}{t}=\left\langle x^{*}, y\right\rangle, \quad \forall y \in X .
$$


称 Banach 空间 $X$ 为一致光滑的, 如果当 $\tau \rightarrow 0^{+}$时, $\frac{\rho(\tau)}{\tau} \rightarrow 0$, 其中 $\rho$ 定义为

$$
\rho(\tau)=\sup \left\{\frac{\|x+\tau y\|+\|x-\tau y\|-2}{2}: x, y \in S_{X}\right\}, \quad \tau \in(0, \infty) .
$$

光滑和一致光滑的 Banach 空间都可以利用其有限维子空间的球覆盖来进行刻画.

定理 4.3 ${ }^{[29]}$ Banach 空间 $X$ 为 Gâteaux 光滑的当且仅当对于任意 $n \in \mathbb{N}$, 任意满足 $\left\langle x_{j}^{*}, x_{j}\right\rangle=1$ 的 $\left\{x_{j}^{*}\right\}_{j=0}^{n} \subset S_{X^{*}}$ 和 $\left\{x_{j}\right\}_{j=0}^{n} \subset S_{X}$, 以及任意被 $\left\{x_{j}^{*}\right\}_{j=0}^{n}$ 正分离的包含 $\left\{x_{j}\right\}_{j=0}^{n}$ 的子空间 $Y$, 存在 $S_{Y}$ 的球覆盖 $\left\{B\left(y_{j}, r_{j}\right)\right\}_{j=0}^{n}$, 其中对于任意 $0 \leqslant j \leqslant n$ 都有 $y_{j} \in \mathbb{R}^{+} x_{j}$.

对于 $1 \geqslant \alpha>0$, 称有界集 $A \subset X^{*}$ 为 $\alpha$-赋范集, 若有

$$
\alpha\|x\| \leqslant \sigma_{A}(x) \equiv \sup _{x \in A}\left\langle x^{*}, x\right\rangle \leqslant \alpha^{-1}\|x\|, \quad x \in X,
$$

其中 1 - 赋范集也称为赋范集.

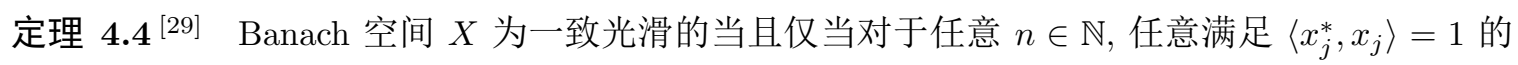
$\left\{x_{j}^{*}\right\}_{j=0}^{n} \subset S_{X^{*}}$ 和 $\left\{x_{j}\right\}_{j=0}^{n} \subset S_{X}$, 以及包含 $\left\{x_{j}\right\}_{j=0}^{n}$ 并以 $\left\{x_{j}^{*}\right\}_{j=0}^{n}$ 为其 $\alpha$-赋范集的子空间 $Y$ 和 $Y$ 都 有球覆盖 $\left\{B\left(y_{j},\left\|y_{j}\right\|-\alpha / 2\right)\right\}_{j=0}^{n}$, 其中对于任意 $0 \leqslant j \leqslant n$ 都有 $y_{j} \in \mathbb{R}^{+} x_{j}$.

称 Banach 空间 $X$ 为一致非方的, 若存在 $\varepsilon>0$ 使得对于任意 $x, y \in S_{X}$ 都有 $\min \|x \pm y\| \leqslant 1-\varepsilon$, 这等价于 $\ell_{1}^{2}$ 不能在 $X$ 中表示. 关于一致非方的 Banach 空间, 文献 [30] 有如下结果:

定理 4.5 Banach 空间 $X$ 为一致非方的当且仅当存在两个整数 $\alpha$ 和 $\beta$, 使得对于 $X$ 中的任意 二维子空间 $U$, 存在 $S_{U}$ 的球覆盖 $\mathcal{B}_{U}$ 满足 $\mathcal{B}_{U}^{\sharp}=3, \mathcal{B}_{U}$ 为 $\alpha$ - 远离原点且 $r\left(\mathcal{B}_{U}\right) \leqslant \beta$.

称 Banach 空间 $X$ 为万有有限表示空间, 如果任意 Banach 空间都能在 $X$ 中有限表示. 例如, $c_{0}$ 和由 $n$ 维空间 $\ell_{n}^{n}$ 的 $\ell_{2^{-}}$和所构成的自反空间 $\left(\sum_{n \geqslant 1} \oplus l_{n}^{n}\right)_{2}$ 就是这样的万有空间.

关于万有有限表示空间, 文献 [31] 中有如下刻画:

定理 4.6 ${ }^{[31]}$ Banach 空间 $X$ 非万有有限表示空间当且仅当存在 $n \geqslant 2$ 和 $\alpha, \beta>0$, 满足对于 $X$ 的任意 $n$ 维子空间 $Y$, 存在 $S_{Y}$ 的最小球覆盖 $\mathcal{B}$ 使得下述成立:

(i) $\mathcal{B}^{\#} \leqslant 2 n-1$;

(ii) $r(\mathcal{B}) \leqslant \beta$;

(iii) $\mathcal{B}$ 为 $\alpha$ - 远离原点.

下面介绍 B- 凸的概念. 1962 年, Beck ${ }^{[32]}$ 首先引入 B- 凸来刻画关于取值于 Banach 空间的随机 变量的强大数定律的存在性.

称 Banach 空间 $X$ 为 B- 凸的, 如果存在 $n \in \mathbb{N}$ 和 $\varepsilon>0$ 使得对于任意 $\left\{x_{j}\right\}_{j=1}^{n} \subset S_{X}$, 有

$$
\min \left\{\left\|\sum_{j=1}^{n} \theta_{j} x_{j}\right\|: \theta_{j} \in\{ \pm 1\}, 1 \leqslant j \leqslant n\right\}<n-\varepsilon,
$$

或者等价地, $\ell_{1}^{n}$ 在 $X$ 中不能有限表示.

对于 B- 凸空间, 有如下结论:

定理 4.7 [31] Banach 空间 $X$ 为 B- 凸的当且仅当存在 $n \in \mathbb{N}$ 和 $\alpha, \beta>0$ 使得对于 $X$ 的任意 $n$ 维子空间 $Y$, 存在 $X^{*} / Y^{0}\left(Y^{0}=\left\{x^{*} \in X^{*}:\left\langle x^{*}, x\right\rangle=0, \forall x \in Y\right\}\right)$ 的最小球覆盖 $\mathcal{B}$ 满足

(i) $\mathcal{B}^{\#} \leqslant 2 n-1$;

(ii) $r(\mathcal{B}) \leqslant \beta$;

(iii) $\mathcal{B}$ 为 $\alpha$ - 远离原点. 
球拓扑概念由 Godefroy 和 Kalton ${ }^{[4]}$ 提出, 它的产生源于以下两个一般性的问题. 第一个问题是 Banach 空间 $X$ 在什么条件之下唯一地等距于一个对偶空间. 称 $X$ 具有唯一的预对偶, 如果存在唯一 的闭子空间 $E \subset X^{*}$ 使得 $X$ 可以看成与 $E^{*}$ 相等. 也可以对 $X$ 具有唯一预对偶的条件换个说法, 即 $X$ 具有唯一的预对偶, 若在 $B_{X}$ 上存在唯一的紧 Hausdorff 拓扑, 这个紧 Hausdorff 拓扑是由 $X$ 上的 某个局部凸线性拓扑诱导而得的.

第二个问题是 Banach 空间 $X$ 在什么条件下决定了其上任意的 Hausdorff 线性拓扑 $\tau$, 满足只要 它使得 $B_{X}$ 是 $\tau$ - 紧的, 就有 $\left.\tau\right|_{B_{X}}$ 与 $X$ 上某个局部凸线性拓扑相等. 这个问题是受到 "Krein-Milman 定理是否在非局部凸 $F$ - 空间上成立?” 这个问题的启发而提出的. 定义点 $x_{0} \in X$ 的 $b_{X^{-}}$邻域基为

$$
V=X \backslash \bigcup_{j=1}^{n} \bar{B}\left(x_{j}, r_{j}\right),
$$

其中 $n \in \mathbb{N}$ 且 $x_{1}, x_{2}, \ldots, x_{n} \in X$ 满足 $\left\|x_{0}-x_{j}\right\|>r_{j}$. 所生成的最粗拓扑即为球拓扑, 具有如下的性 质 (参见文献 $[4$, 第 197 页]):

(1) 对固定的 $y \in X$, 映射 $x \rightarrow x+y$ 为 $b_{X^{-}}$连续的;

(2) 对固定的 $\lambda>0$, 映射 $x \rightarrow \lambda x$ 为 $b_{X^{-}}$连续的;

(3) 映射 $x \rightarrow-x$ 为 $b_{X^{-}}$连续的.

称拓扑空间中点 $x_{0}$ 为 $G_{\delta}$ 的, 如果存在 $x_{0}$ 的开邻域序列 $\left\{V_{n}\right\}$ 使得 $\left\{x_{0}\right\}=\bigcap_{n \in \mathbb{N}} V_{n}$. 于是有下 面的定理:

定理 $4.8^{[29]}$ 在赋予球拓扑 $b_{X}$ 的 Banach 空间 $X$ 中, 每个点均为 $\left(b_{X}-G_{\delta}\right)$ - 点当且仅当 $X$ 具有 球覆盖性质. 所以, 具有球覆盖性质的 Banach 空间中 $b_{X^{-}}$紧集均为 $b_{X^{-}}$序列紧的.

注 4.1 由于球覆盖性质关于线性同胚并不保持 (见下一节), 所以, Banach 空间 $X$ 中点的 $\left(b_{X}-G_{\delta}\right)$ - 性质并不是拓扑不变的.

此外, 王波 ${ }^{[33]}$ 证明了, 如果 $X$ 具有 $w^{*}$ - 可分对偶且满足 Mazur 交性质, 则 $X$ 具有球覆盖性质.

\section{5 球覆盖性质的稳定性问题}

尽管球覆盖性质可以刻画 Banach 空间中许多有趣的几何性质和拓扑性质, 但是要注意球覆盖性 质也有不足之处, 例如, 关于线性同胚不保持, 并且不具有关于闭子空间的遗传性等.

这里假设 $X=\ell_{\infty}$, 则商空间 $\ell_{\infty} / c_{0}$ 的范数为 $\|x\|_{Q} \equiv \lim \sup _{n}|x(n)|, \forall x=(x(n)) \in \ell_{\infty}$.

对于给定的 $0<\lambda \leqslant 1$, 设 $\|x\|_{\lambda}=\lambda\|x\|+(1-\lambda)\|x\|_{Q}, \forall x \in \ell_{\infty}$, 则 $\|\cdot\|_{\lambda}$ 为 $\ell_{\infty}$ 的等价范数.

关于这样的空间, 有下面的定理:

定理 $5.1^{[34]}$ (i) $\left(\ell_{\infty},\|\cdot\|_{\lambda}\right)$ 具有球覆盖性质当且仅当 $1 \geqslant \lambda>\frac{1}{2}$;

(ii) 商空间 $\ell_{\infty} / c_{0}$ 不具有球覆盖性质.

定理 $5.2^{[27]}$ (i) $\ell_{1}[0,1]$ 不具有球覆盖性质;

(ii) $\ell_{\infty}$ 具有球覆盖性质;

(iii) $\ell_{1}[0,1]$ 线性同胚于 $\ell_{\infty}$ 的子空间.

最近, Luo 和 Zheng ${ }^{[35]}$ 给出了对于一列 Banach 空间, 球覆盖性质关于 $\ell_{p^{-}}$和保持, 其中 $1 \leqslant p \leqslant \infty$. 接下来将用完全不同的方法给出较之更为广泛的有关结果.

设 $s=\left\{x=(x(n))_{n=1}^{\infty}, x(n) \in \mathbb{R}\right\}$ 为 Banach 序列空间, 当 $m=n$ 时, $e_{n}=\delta_{m, n}=1$; 当 $m \neq n$ 时, $e_{n}=\delta_{m, n}=0$. 对于 $x=(x(n)) \in s, e_{n}^{*}$ 定义为 $\left\langle e_{n}^{*}, x\right\rangle=x(n), n=1,2, \ldots$ 称 $s$ 单调, 如果对于任意 
$n \in \mathbb{N}$ 都有 $\left\|e_{n}\right\|=1=\left\|e_{n}^{*}\right\|$, 以及任意满足 $a_{n} \in \mathbb{R}$ 的序列 $\left(a_{n}\right)_{n=1}^{\infty}$,

$$
\left\|\sum_{j=1}^{n} a_{j} e_{j}\right\| \leqslant\left\|\sum_{j=1}^{n+1} a_{j} e_{j}\right\|, \quad \forall n \in \mathbb{N} ;
$$

称单调的 $s$ 为严格单调, 如果对于任意满足 $a_{n}, b_{n} \in \mathbb{R}^{+}$和 $b_{j} \geqslant a_{j}(1 \leqslant j \leqslant n)$ 的序列 $a=\left(a_{j}\right)_{j=1}^{n}$ 和 $b=\left(b_{j}\right)_{j=1}^{n}, b \neq a$, 都有

$$
\left\|\sum_{j=1}^{n} a_{j} e_{j}\right\|<\left\|\sum_{j=1}^{n} b_{j} e_{j}\right\| .
$$

容易知道, 具有 1- 无条件基的 Banach 空间线性同胚于单调序列空间. 称 $s$ 为坐标光滑, 如果 $e_{n}$ $(n \in \mathbb{N})$ 为 $s$ 中关于范数的 Gâteaux 可微点. 例如, $\ell_{p}(1<p \leqslant \infty)$ 和 $c_{0}$ 单调且坐标光滑, 而 $\ell_{p}$ $(1 \leqslant p<\infty)$ 为严格光滑的.

对于列 Banach 空间 $\left(X_{n}\right),\left(X_{n}\right)$ 的 $s$ - 和定义为

$$
X \equiv\left(\bigoplus_{n \in \mathbb{N}} X_{n}\right)_{s}=\left\{x=\left(x_{1}, x_{2}, \ldots, x_{n}, \ldots\right): x_{n} \in X_{n}, n \in \mathbb{N}\right\},
$$

在上面赋予范数 $\|x\|=\left\|\left(\left\|x_{1}\right\|,\left\|x_{2}\right\|, \ldots\right)\right\|_{s}$.

首先对于单调且坐标光滑的 Banach 序列空间, 结果如下:

定理 5.3 设 $s$ 为坐标光滑的单调 Banach 序列空间, $\left\{X_{n}\right\}$ 为一列 Banach 空间, 而 $X \equiv$ $\left(\bigoplus_{n \in \mathbb{N}} X_{n}\right)_{s}$, 则空间 $X$ 的单位球面 $S_{X}$ 具有满足对于任意 $m \in \mathbb{N}$ 都有 $\left\{x_{m n}\right\}_{n=1}^{\infty} \in X_{m}$ 的球覆 盖 $\mathcal{B}=\left\{B\left(x_{m n}, r_{m n}\right): m, n \in \mathbb{N}\right\}$, 当且仅当每个 $X_{n}$ 都具有球覆盖性质.

证明 充分性. 设 $X_{m}=\left(X_{m},\|\cdot\|_{m}\right)(m \in \mathbb{N})$ 具有球覆盖性质, 则对于任意给定 $m \in \mathbb{N}$, 存在 $S_{m} \equiv S_{X_{m}}$ 的球覆盖 $\mathcal{B}_{m}=\left\{B\left(x_{m n, r_{m n}}\right)\right\}_{n=1}^{\infty}$. 由定理 1.1 可得, 这等价于对于次微分映射 $\partial\|\cdot\|_{m}$ 的任 意选择 $\varphi_{m},\left\{\varphi_{m}\left(x_{m n}\right)\right\}$ 正分离了 $X_{m}$ 中的点. 因为 $\pm e_{m}$ 为 $s$ 的光滑点, $\partial\left\|e_{m} x_{m n}\right\|_{X}=e_{m} \partial\left\|x_{m n}\right\|_{m}$, 其中, 当 $n=m$ 时, $X \ni e_{m} x_{m n}=x_{m m}$; 当 $n \neq m$ 时, $X \ni e_{m} x_{m n}=0$. 这说明对于次微分映射 $\partial\|\cdot\|_{X}$ 的任意选择 $\varphi$, 双下标序列 $\left\{\varphi\left(x_{m n}\right)\right\}$ 正分离 $X$ 中的点. 再根据定理 1.1 即完成证明.

必要性. 设 $X$ 具有球覆盖 $\mathcal{B}=\left\{B\left(x_{m n}, r_{m n}\right): m, n \in \mathbb{N}\right\}$, 其中对于任意 $m \in \mathbb{N}$ 都有 $\left\{x_{m n}\right\}_{n=1}^{\infty}$ $\in X_{m}$, 则对于任意给定 $m \in \mathbb{N}, \mathcal{B}_{m}=\left\{B\left(x_{m n}, r_{m n}\right) \cap X_{m}: n \in \mathbb{N}\right\}$ 也是 $X_{m}$ 的球覆盖.

推论 5.1 ${ }^{[35]}$ 设 $s \in\left\{\ell_{p}, c_{0}: 1<p \leqslant \infty\right\}$, 且 $\left\{X_{n}\right\}$ 为一列 Banach 空间, 则空间 $X \equiv\left(\bigoplus_{n \in \mathbb{N}} X_{n}\right)_{s}$ 的球面 $S_{X}$ 具有满足对于任意 $m \in \mathbb{N}$ 都有 $\left\{x_{m n}\right\}_{n=1}^{\infty} \in X_{m}$ 的球覆盖 $\mathcal{B}=\left\{B\left(x_{m n}, r_{m n}\right): m, n \in \mathbb{N}\right\}$, 当且仅当每个 $X_{n}$ 都具有球覆盖性质.

对于严格单调的可分 Banach 序列空间, 则有下面的定理:

定理 5.4 设 $s$ 为严格单调的可分 Banach 序列空间, $\left\{X_{n}\right\}$ 为一列 Banach 空间, 而 $X \equiv$ $\left(\bigoplus_{n \in \mathbb{N}} X_{n}\right)_{s}$, 则空间 $X$ 的球面 $S_{X}$ 具有球覆盖当且仅当每个 $X_{n}$ 都具有球覆盖性质.

证明 充分性. 因为 $s$ 可分, 所以, $s$ 为 Gâteaux 可微空间. 根据推论 4.1, 存在满足对于任意 $n \in \mathbb{N}$ 都有 $d\left\|s_{n}\right\|=s_{n}^{*}$ 的序列 $\left\{s_{n}\right\} \subset S_{s}$ 和 $\left\{s_{n}^{*}\right\} \subset S_{s^{*}}$, 使得 $\left\{s_{n}^{*}\right\}$ 正分离 $s$ 中的点. 因为 $X_{n}(n=1,2, \ldots)$ 都具有球覆盖性质, 根据定理 1.1 , 对任意给定 $m \in \mathbb{N}$, 存在序列 $\left\{x_{m n}\right\}_{n=1}^{\infty} \subset S_{\left(X_{m},\|\cdot\|_{m}\right)}$ 使得对于范 数 $\|\cdot\|_{m}$ 的次微分映射 $\partial\|\cdot\|_{m}$ 的任意选择 $\varphi_{m},\left\{\varphi_{m}\left(x_{m n}\right)\right\}$ 正分离 $X_{m}$ 中的点. 令 $s_{n}=\left(s_{n}(j)\right)$ 且 $d\left\|s_{n}\right\|\left(=s_{n}^{*}\right)=\left(s_{n}^{*}(j)\right)$, 则

$$
1=\left\langle s_{n}^{*}, s_{n}\right\rangle=\sum_{j=1}^{\infty} s_{n}^{*}(j) \cdot s_{n}(j)=\sum_{j=1}^{\infty}\left|s_{n}^{*}(j) \cdot s_{n}(j)\right| .
$$


现在取

$$
x_{n}=s_{n} \cdot\left(x_{m n}\right)=\left(\bigoplus_{m=1}^{\infty} s_{n}(m) x_{m n}\right)_{s} \in X,
$$

则对于任意 $n \in \mathbb{N}$, 有 $\left\|x_{n}\right\|=\left\|s_{n}\right\|=1$, 且

$$
\partial\left\|x_{n}\right\|=\left\{\left(\bigoplus_{m=1}^{\infty} s_{n}^{*}(m) x_{m n}^{*}\right)_{s^{*}}: x_{m n}^{*} \in \partial\left\|x_{m n}\right\|, m, n \in \mathbb{N}\right\} .
$$

这说明 $\partial\left\|x_{n}\right\|=\left(\bigoplus_{m=1}^{\infty} s_{n}^{*}(m) \partial\left\|x_{m n}\right\|\right)_{s^{*}}$. 也就是说, 对于 $\partial\|\cdot\|_{X}$ 的任意选择 $\varphi$, 存在一列 $\partial\|\cdot\|_{m}$ 的 选择 $\varphi_{m}$, 使得 $\varphi\left(x_{n}\right)=\left(\bigoplus_{m=1}^{\infty} s_{n}^{*}(m) \varphi\left(x_{m n}\right)\right)_{s^{*}} \cdot\left\{\varphi\left(x_{n}\right)\right\}$ 正分离了 $X$ 中的点.

必要性. 若结论不成立, 则假设对于某个 $m \in X, X_{m}$ 不具有球覆盖, 则对于任意序列 $\left(x_{m n}\right) \subset$ $S_{m} \equiv S_{X_{m}}$, 存在 $\partial\|\cdot\|_{m}$ 的选择 $\varphi_{m}$ 使得 $\left\{\varphi_{m}\left(x_{m n}\right)\right\}$ 不能正分离 $X_{m}$ 中的点. 根据 (5.1) 可知, 对于 任意序列 $\left\{x_{n}\right\} \subset S_{X}$, 存在 $\|\cdot\|$ 的选择 $\varphi$ 使得 $\left\{\varphi\left(x_{n}\right)\right\}$ 不能正分离 $X$ 的点.

推论 5.2 [35] 设 $s=\ell_{1},\left\{X_{n}\right\}$ 为一列 Banach 空间, 则空间 $X \equiv\left(\bigoplus_{n \in \mathbb{N}} X_{n}\right)_{s}$ 的球面 $S_{X}$ 具有球 覆盖性质当且仅当每个 $X_{n}$ 都具有球覆盖性质.

值得注意的是, 对于有限测度空间, 有如下结果:

定理 5.5 [35] 设 $(\Omega, \Sigma, \mu)$ 为有限测度空间, $X$ 为赋范空间, 则 $L_{p}(\mu, X)(1 \leqslant p<+\infty)$ 具有球覆 盖性质当且仅当 $X$ 具有球覆盖性质.

然而, $L_{\infty}[0,1]$ 不具有球覆盖性质.

注 5.1 Shang ${ }^{[36]}$ 及 Shang 和 Cui ${ }^{[37-39]}$ 研究了球覆盖性质并得到许多有趣的结果. 特别的是, Shang ${ }^{[36]}$ 首先讨论了球覆盖性质关于 $\ell_{p^{-}}$和空间 $X \oplus_{\ell_{p}} Y(1 \leqslant p \leqslant+\infty)$ 的遗传性. 利用推论 4.1 , 他 证明了, 若 $X$ 和 $Y$ 为 Gâteaux 可微空间, 则 $X \oplus_{\ell_{p}} Y$ 具有球覆盖性质当且仅当 $X$ 和 $Y$ 都具有球覆 盖性质. 通过直接构造可数球覆盖, Luo 等 ${ }^{[40]}$ 进一步证明了 Shang ${ }^{[36]}$ 的结果中 “Gâteaux 可微性空 间”的假设是可以去掉的.

\section{6 尚待解决的问题}

目前为止, 虽然已经知道 Banach 空间具有球覆盖性质当且仅当 $X^{*}$ 在再赋范的意义下 $w^{*}$ - 可分, 我们还不知道是否存在不可分的 Banach 空间可以在线性同胚的意义下保持球覆盖性质. 所以, 我们 的问题分为两个部分: 一部分问题期待肯定解决, 而另一部分则倾向否定答案.

\section{1 期待肯定解决的问题}

由前文所述, 已经知道球覆盖性质关于线性同胚不保持, 且 Banach 空间的球覆盖性质关于子空 间不具有遗传保持性 (定理 5.1 和 5.2). 然而, 所有与之有关的反例都不是 Asplund 空间, 甚至都不是 Gâteaux 可微空间. 所以, 自然有下述问题:

问题 6.1 除了自反空间外, 对于怎样的 Banach 空间, 其具有的球覆盖性质可以关于线性同胚 保持?

问题 6.2 是否对于 Asplund 空间, 或者更一般的 Gâteaux 可微空间, 其具有的球覆盖性质可以 关于线性同胚保持?

问题 6.3 对于哪一类 Banach 空间, 其具有的球覆盖性质可以关于商映射保持?

问题 6.4 对于怎样的 Banach 空间, 其具有的球覆盖性质可以关于闭子空间遗传? 
注意到对于 Banach 空间 $X$, 如果用 $(\Omega, \rho)$ 来表示 $X$ 上所有连续半范数 $p$, 并且在 $X$ 上面赋予如 下定义的度量 $\rho$ : 对于 $p_{1}, p_{2} \in \Omega$, 有 $\rho\left(p_{1}, p_{2}\right)=\sup _{x \in B_{X}}\left|p_{1}(x)-p_{2}(x)\right|$, 则 $\Omega$ 为完备度量空间, 并且 $X^{*}$ 的 $w^{*}$ - 可分性所推导出的 $X$ 所有具有球覆盖性质的等价范数在 $\Omega$ 中稠密. 进一步有下面的问题:

问题 6.5 对于不可分的具有 $w^{*}$ - 可分对偶的 Banach 空间 $X, X$ 所有具有球覆盖性质的等价 范数是否为 $\Omega$ 中稠 $G_{\delta}$ 子集?

\section{2 倾向否定答案的问题}

因为 $\ell_{1}^{*}[0,1]=\ell_{\infty}[0,1]$ 是 $w^{*}$ - 可分的且不具有球覆盖性质, 但 $\ell_{1}[0,1]$ 又具有 Radon-Nikodým 性 质, 所以, 并不是所有具有 $w^{*}$ - 可分对偶, 同时, 满足 Radon-Nikodým 性质的 Banach 空间都可以使其 具有的球覆盖性质关于线性同胚保持不变. 于是提出下面的问题:

问题 6.6 若具有球覆盖性质的 Banach 空间满足其球覆盖性质关于线性同胚保持不变, 是否 $X$ 一定可分?

最后, 值得关注的是, Papini ${ }^{[41]}$ 研究了有关单位球或单位球面的各种覆盖问题, 这其中就包含了 不含原点的球覆盖. 文献 [42] 对覆盖 $L_{p}$ 的球族进行了讨论, 文献 [43] 还研究了覆盖特定 Banach 空 间的球族和切片族的性质.

本文中的定理 5.3 和 5.4 为新的结果, 其余结果的证明则基于相应参考文献中的证明.

致谢 作者感谢所有专家对本文所提供的意见和建议, 同时本文得益于厦门大学泛函分析团队的探讨和支持, 在此表 示感谢.

\section{参考文献}

1 Akhmerov R R, Kamenskii M I, Potapov A S, et al. Measures of Noncompactness and Condensing Operators. Basel: Birkhäuser, 1992

2 Appell J. Recent Trends in Nonlinear Analysis. Basel: Birkhäuser, 2000

3 Enflo P. Banach spaces which can be given an equivalent uniformly convex norm. Israel J Math, 1972, 13: 281-288

4 Godefroy G, Kalton N J. The ball topology and its applications. In: Contemporary Mathematics, vol. 85. Providence: Amer Math Soc, 1989, 195-237

5 James R C. Uniformly non-square Banach spaces. Ann of Math (2), 1964, 80: 542-550

6 Pełczyński A. All separable Banach space admit for every $\varepsilon>0$ fundamental total and bounded by $1+\varepsilon$ biorthogonal sequences. Studia Math, 1976, 55: 295-304

7 Vanderwerff J. Mazur intersection properties for compact and weakly compact convex sets. Canad Math Bull, 1998, 41: $225-230$

8 Zizler V. Renorming concerning Mazur's intersection property of balls for weakly compact convex sets. Math Ann, 1986, 276: $61-66$

9 Kuratowski K. Sur les espaces complets. Fund Math, 1930, 15: 301-309

10 Darbo G. Punti uniti in trasformazioni a codominio non compatto. Rend Sem Mar Univ Padova, 1955, 24: 84-92

11 Goldenstein L S, Gochberg I T, Markus A S. Investigation of some propertied of bounded linear operators in connection with their q-norms. Učen Zap Kishinevsk Univ, 1957, 29: 29-36

12 Cheng L. Ball-covering property of Banach spaces. Israel J Math, 2006, 156: 111-123

13 Fonf V P, Zanco C. Covering spheres of Banach spaces by balls. Math Ann, 2009, 344: 939-945

14 Phelps R R. Convex Functions, Monotone Operators and Differentiability. Lecture Notes in Mathematics, vol. 1364. New York: Springer-Verlag, 1989

15 Cheng L, Shi H, Zhang W. Every Banach space with a $w^{*}$-separable dual has a $1+\varepsilon$-equivalent norm with the ball covering property. Sci China Ser A, 2009, 52: 1869-1874

16 Cheng L, Cheng Q, Shi H. Minimal ball-coverings in Banach spaces and their application. Studia Math, 2009, 192: $15-27$

17 刘根洪. $E^{n}$ 中 $n$ 维单形外接超球面的半径. 苏州大学学报 (自然科学版), 1990, 6: 1-5

18 张㿟晶. $R^{n}$ 空间中单位球面的覆盖问题. 数学研究, 2007, 40: 109-113 
19 林国琛, 沈喜生. 计算球覆盖最小半径的神经网络方法. 厦门大学学报 (自然科学版), 2008, 47: 797-800

20 Gerber L. The orthocentric simplex as an extreme simplex. Pacific J Math, 1975, 56: 97-111

21 傅瑞瑜, 程立新. Banach 空间单位球面的球覆盖性质. 数学研究, 2006, 39: 39-43

22 林丽华. 单位球极小球覆盖数为 $n+1$ 的 $n$ - 维赋范空间的性质. 淮北煤炭师范学院学报 (自然科学版), 2008, 29: 18-22

23 林丽华, 张风, 张敏. $n$ - 维赋范空间单位球极小球覆盖数 $2 n-1$ 与包含等距同构于 $\left(\mathbb{R}^{n-1},\|\cdot\|_{\infty}\right)$ 的子空间. 数学 研究, 2008, 41: 407-415

24 施慧华, 张㿟晶. $R^{n}$ 空间中单位球面的极小球覆盖. 厦门大学学报 (自然科学版), 2006, 45: 621-623

25 林丽华. 遗传不可分解空间与具有球覆盖性质空间的关系. 华东师范大学学报 (自然科学版), 2008, 3: 8-11

26 Bishop E, Phelps R R. A proof that every Banach space is subreflexive. Bull Amer Math Soc (NS), 1961, 67: 97-98

27 Cheng L, Kadets V, Wang B, et al. A note on ball-covering property of Banach spaces. J Math Anal Appl, 2010, 371: 249-253

28 Guirao A J, Lissitsin A, Montesinos V. Some remarks on the ball-covering property. J Math Anal Appl, 2019, 479: $608-620$

29 Cheng L, Wang B, Zhang W, et al. Some geometric and topological properties of Banach spaces via ball coverings. J Math Anal Appl, 2011, 377: 874-880

30 Cheng L, Luo Z, Liu X, et al. Several remarks on ball-coverings of normed spaces. Acta Math Sin Engl Ser, 2010, 26: $1667-1672$

31 Zhang W. Characterizations of universal finite representability and B-convexity of Banach spaces via ball coverings. Acta Math Sin Engl Ser, 2012, 28: 1369-1374

32 Beck A. A convexity condition in Banach spaces and the strong law of large numbers. Proc Amer Math Soc, 1962, 13: 329-334

33 王波. 两类赋予 $w^{*}$ - 可分对偶的空间的 BCP. 数学研究, 2010, 43: 393-396

34 Cheng L, Cheng Q, Liu X. Ball-covering property of Banach spaces that is not preserved under linear isomorphisms. Sci China Ser A, 2008, 51: 143-147

35 Luo Z, Zheng B. Stability of the ball-covering property. Studia Math, 2020, 250: 19-34

36 Shang S. Differentiability and ball-covering property in Banach spaces. J Math Anal Appl, 2016, 434: 182-190

37 Shang S, Cui Y. Ball-covering property in uniformly non- $l_{3}^{1}$ Banach spaces and application. Abstr Appl Anal, 2013 , 2013: $1-7$

38 Shang S, Cui Y. Locally 2-uniform convexity and ball-covering property in Banach space. Banach J Math Anal, 2015 , 9: $42-53$

39 Shang S, Cui Y. Dentable point and ball-covering property in Banach spaces. J Convex Anal, 2018, 25: 1045-1058

40 Luo Z, Liu J, Wang B. A remark on the ball-covering property of product spaces. Filomat, 2017, 31: 3905-3908

41 Papini P L. Covering the sphere and the ball in Banach spaces. Commun Appl Anal, 2009, 13: 579-586

42 Fonf V P, Levin M, Zanco C. Covering $L^{p}$ spaces by balls. J Geom Anal, 2014, 24: 1891-1897

43 Fonf V P, Zanco C. Covering the unit sphere of certain Banach spaces by sequences of slices and balls. Canad Math Bull, 2014, 57: 42-50

\section{Ball-covering property of Banach spaces}

\section{Wen Zhang}

Abstract A Banach space admitting a unit sphere covering consisting of countably many balls off the origin is said to have ball-covering property. In this paper, we present a brief review on the research area of ball-covering property of Banach spaces, and some new results about this topic are included.

Keywords Banach space, ball-covering property, subdifferential mapping

MSC(2010) 46B20

doi: $10.1360 /$ SSM-2020-0062 